\title{
Axisymmetrical gas inflow in the central region of NGC 7331
}

\author{
E. Battaner ${ }^{1}$, E. Mediavilla², A. Guijarro ${ }^{3}$, S. Arribas ${ }^{4}$, and E. Florido ${ }^{1}$ \\ 1 Dpto. Física Teórica y del Cosmos, Universidad de Granada, Spain \\ 2 Instituto de Astrofísica de Canarias, Tenerife, Spain \\ 3 Centro Astronómico Hispano Alemán, Almería, Spain \\ ${ }^{4}$ Space Telescope Science Institute, Baltimore, USA. Affiliated with the RSS Department of the European Space Agency. \\ On leave from the IAC - CSIC
}

Received 11 March 2002 / Accepted 4 July 2002

\begin{abstract}
New Integral Field Spectroscopy of the central region of NGC 7331 reveals strong H $\alpha$ emission in the well-known $\mathrm{CO}$ and $\mathrm{HI}$ ring of $\mathrm{NGC} 7331$. The $[\mathrm{NII}] / \mathrm{H} \alpha$ ratio indicates that a large scale stellar formation process is taken place at the ring in agreement with previous hypothesis about the exhaustion of gas in the inner to the ring region. The dynamics of stars and gas are not coupled. There is a ring of peculiar velocities in the ionized gas velocity map. These peculiar velocities can be well interpreted by the presence of an axisymmetric inflow of $40 \mathrm{~km} \mathrm{~s}^{-1}$ at the inner boundary of the large-scale gaseous ring. We infer an inwards total flux of $1.6 M_{\odot} \mathrm{yr}^{-1}$. This value is typical of the accretion rates in hypothetical large nuclear black holes. Despite the large differences in the scales of the nucleus and the gas ring of NGC 7331, we suggest that this inwards flux is feeding the nucleus.
\end{abstract}

Key words. galaxies: active - galaxies: individual: NGC 7331 - galaxies: kinematics and dynamics - galaxies: spiral

\section{Introduction}

Peculiar motions are frequently detected in active galaxies where the existence of several kinematically distinct gaseous systems, some of them suffering radial outward movements, has been attributed to the influence of the active nucleus (García-Lorenzo et al. 1999, 2001; Arribas et al. 1997; Mediavilla \& Arribas 1993). The outflow of gas close to the nucleus is relatively easy to detect because of the large velocities involved (of the order of $100 \mathrm{~km} \mathrm{~s}^{-1}$ ) and of the direct illumination from the strong active source. Inflow of gas seems to be much more difficult to observe as it is presumably caused by less outstanding mechanisms than the nuclear activity. However, it is very important to detect since inflow should provide the matter that will eventually accrete into the nuclear black hole. To study the influence of the environment on the nucleus we will present new results about the kinematics in the central region of NGC 7331. Some peculiarities previously detected in the ionized gas velocity field of this galaxy (see below) make it specially interesting for this kind of studies.

The large scale gas distribution in NGC 7331 is ring-like as first detected by Bosma (1978) in HI, Telesco et al. (1982) from NIR colors and Young \& Scoville (1982) in CO. The ring is confirmed in other wavelengths and is taken today as a prototype gas ring.

Bower et al. (1993) used optical long-slit spectra to show that their models without a central black hole fit the

Send offprint requests to: E. Battaner, e-mail: battaner@ugr.es observational data. However, there is an unresolved LINER nucleus (see, for instance, Cowan et al. 1994), so this galaxy could harbour a massive black hole of $5 \times 10^{8} M_{\odot}$. This is supported by the motion of ionized gas ([NII] $+\mathrm{H} \alpha$ emission line) reported by Afanasiev et al. (1989) in the 0.2-0.4 kpc $(2.8-5.6$ arcsec) zone and by the discovery of a nuclear X-ray source by Stockdale et al. (1998) using ROSAT.

The existence of a bar has been suggested from non-circular motions by Marcelin et al. (1994) and von Linden et al. (1996). However the $I$ and $K$ band photometry in the central regions carried out by Prada et al. (1996) did not indicate any significant barred morphology.

In the central kpc, Bottema (1999) found that the emission line gas (for $R \leq 40 \mathrm{arcsec}$ ) seems to rotate slower than the stars. An explanation of the observations would consist in an inclined and warped gaseous plane.

Using 2D spectroscopy, Mediavilla et al. (1997) concluded that the kinematics of the stars and most of the ionized gas are decoupled. These authors found that the kinematic axes of the ionized gas velocity map are distorted and rotated with respect to the stars, something that can be interpreted in terms of radial movements. However, the reduced field of view of the available velocity maps (about $7^{\prime \prime} \times 7^{\prime \prime}$ ) makes difficult to verify this interpretation.

In this letter we are going to present new Integral Field spectroscopy covering the central $30^{\prime \prime} \times 30^{\prime \prime}$ of NGC 7331, with the aim of understanding the kinematics of the region surrounding the nucleus. 


\section{Observations and reduction}

NGC 7331 was observed with INTEGRAL (Arribas et al. 1998) in combination with the fiber spectrograph WYFFOS (Bingham et al. 1994) the 15 of July of 2001. The spectral resolution and coverage were $4.8 \AA$ and $1445 \AA$ respectively. The central wavelength was $6200 \AA$. From the wavelength calibration we estimate that the uncertainty in velocity determinations is better than $10 \mathrm{~km} \mathrm{~s}^{-1}$. We used the SB3 bundle of INTEGRAL. This bundle consists of a central rectangle of $30^{\prime \prime} \times 34^{\prime \prime}$ and an outer ring of $90^{\prime \prime}$ in diameter. Four exposures of 600s each were taken from the central region of NGC 7331. These frames were combined to increase the $S / N$ ratio and to reject the cosmic rays. Posteriorly, the spectra were extracted, calibrated in wavelength, and corrected from throughput. The reduction steps were made using the INTEGRAL data reduction package running in IRAF. More details about the reduction procedure can be found in Arribas et al. (1991).

For several spectral features of interest we have determined a grid of values at the locations of the fibers at the focal plane and have interpolated a map of the selected feature (see, e.g., Arribas et al. 1999).

In Fig. 1 we show the resulting intensity and velocity maps. The stellar continuum corresponds to the $\sim 5730-6800 \AA$ A, spectral range. The stellar velocity map has been obtained by cross correlating the spectra in the $\sim 5600 \AA-6850 \AA$ wavelength interval using the nuclear spectrum as template. We also present intensity and velocity maps obtained from the multi-Gaussian fit of the $\mathrm{H} \alpha+[\mathrm{NII}] \lambda \lambda 6548,6583$ blend. The nuclear spectrum is very similar to the ones obtained from the same region by Filippenko \& Sargent (1985) or Mediavilla et al. (1997) and shows a strong $\mathrm{H} \alpha$ absorption (Mediavilla et al. give a description of the nuclear spectrum of NGC 7331 in comparison with that of M 31). To avoid the influence of the strong $\mathrm{H} \alpha$ absorption in the determination of the velocity and intensity maps, we have considered in the fits an extra component representing this absorption feature (see Mediavilla et al. 1997, for details).

\section{Observational results}

A very noticeable feature in Fig. 1 is the ring structure observed in the $\mathrm{H} \alpha$ intensity map. The two-dimensional distribution of the $\mathrm{H} \alpha$ emission consists of a nucleus, a region with very low emission and then the $\mathrm{H} \alpha$ ring that begins rather abruptly at about 7 arcsec measured in the east direction $(\sim 500 \mathrm{pc}$ equivalent to a galactocentric radius of about $2 \mathrm{kpc}$, considering $i=75^{\circ}$ ). Therefore we are observing the inner part of the well-known large-scale gaseous ring. In contrast, the [NII] emission decays continuously from the nucleus towards the outer parts and is very faint at the ring location. In the ring the $[\mathrm{NII}] \lambda 6583 / \mathrm{H} \alpha$ ratio is about 0.3 , typical of ionization by a HII-like continuum. This indicates that the ring is representative of a large scale stellar formation process. On the contrary, the $[\mathrm{NII}] \lambda 6583 / \mathrm{H} \alpha$ ratio in the inner to the ring region is very high, about 5 . This is typical of LINERS. At the nucleus we found a value [NII] $\lambda 6583 / \mathrm{H} \alpha \sim 3$ also typical of LINERS and similar to the one (2.7) reported by Mediavilla et al. (1997). It is also remarkable that the transition between the LINER and the starburst regions is very abrupt and takes place at the inner boundary of the $\mathrm{H} \alpha$ ring.

Another important feature in the maps of Fig. 1 is the presence of a symmetrical distortion in the velocity maps corresponding to the ionized gas, also coincident with the inner boundary of the starburst ring.

Leaving aside the S-shaped distortion present in the innermost region (see Mediavilla et al. 1997), the stellar velocity map in NGC 7331 is quite regular. However, the dynamics observed in $\mathrm{H} \alpha$ and [NII] is very much distorted when compared with the pure rotation velocity field of the stellar system. Notice that the central $7^{\prime \prime} \times 7^{\prime \prime}$ of the $\mathrm{H} \alpha$ map (Fig. 1) are in very good agreement with the $\mathrm{H} \alpha$ velocity map presented in Mediavilla et al. (1997).

\section{The large scale inflow}

The new map of $\mathrm{H} \alpha$ velocities (Fig. 1) gives a global perspective of the central region of NGC 7331 and can be interpreted in terms of a ring of peculiar velocities superimposed to a normal rotation pattern. In Fig. 2 we represent the velocity as a function of galactic azimuthal angle, $v(\theta)$, along the ring of peculiar velocities for $\mathrm{H} \alpha$ and the stars. As it can be seen the $\mathrm{H} \alpha$ curve presents a global blueshift of $60 \mathrm{~km} \mathrm{~s}^{-1}$ with respect to the stellar curve (the $[\mathrm{NII}]$ curve, not represented here, suffers also a blueshift of $23 \mathrm{~km} \mathrm{~s}^{-1}$ ). After removing the global blueshift, the $\mathrm{H} \alpha$ curve is shifted by about 25 degrees with respect to the stellar one (see Fig. 2). The [NII] curve corrected from blueshift is intermediate between that of the stars and $\mathrm{H} \alpha$ showing a shift of 15 degrees with respect to the stellar rotation. The amplitudes of the $\mathrm{H} \alpha$ and [NII] curves are 117 and $95 \mathrm{~km} \mathrm{~s}^{-1}$, respectively. Thus, the kinematic shifts with respect to the stellar rotation imply a radial velocity component of about $55 \mathrm{~km} \mathrm{~s}^{-1}$ for $\mathrm{H} \alpha$ and $26 \mathrm{~km} \mathrm{~s}^{-1}$ for [NII]. With an alternative method, measuring the departures of the velocity field from the systemic velocity along the minor kinematic axis, we have obtained a similar value, $45 \mathrm{~km} \mathrm{~s}^{-1}$ for $\mathrm{H} \alpha$.

The west part of this kinematically peculiar ring is relatively redder and the east-part relatively bluer. As the dust lanes in optical images clearly indicate that the west part is the closest, we can conclude that we are observing a contraction motion (see Fig. 3). Thus, we have detected gas flowing from the $\mathrm{H} \alpha$ ring to the central inner region with a very noticeable axisymmetry. The velocity is maximum at the inner boundary of the large-scale gaseous ring. It then decreases towards the center, probably as $V \propto(R \rho)^{-1}$, where $V$ is the inward component of the gas velocity at the inner boundary of the ring, $R$ the galactocentric radius and $\rho$ the density, due to continuity requirements.

Interpretations other than axisymmetric inflow cannot be in principle disregarded to explain the radial motion. An elliptical motion would produce a departure from circular motion, without a net inflow. Also, the observed motions could be attributable to a pair of tightly wound spiral arms, i.e. streaming motions along the spiral arms due to a density wave could produce a similar observed velocity pattern. These effects are not axisymmetric, but as long as axisymmetry is not perfect, they should be considered as alternative interpretations. However, it would be more difficult to account for the observed global 

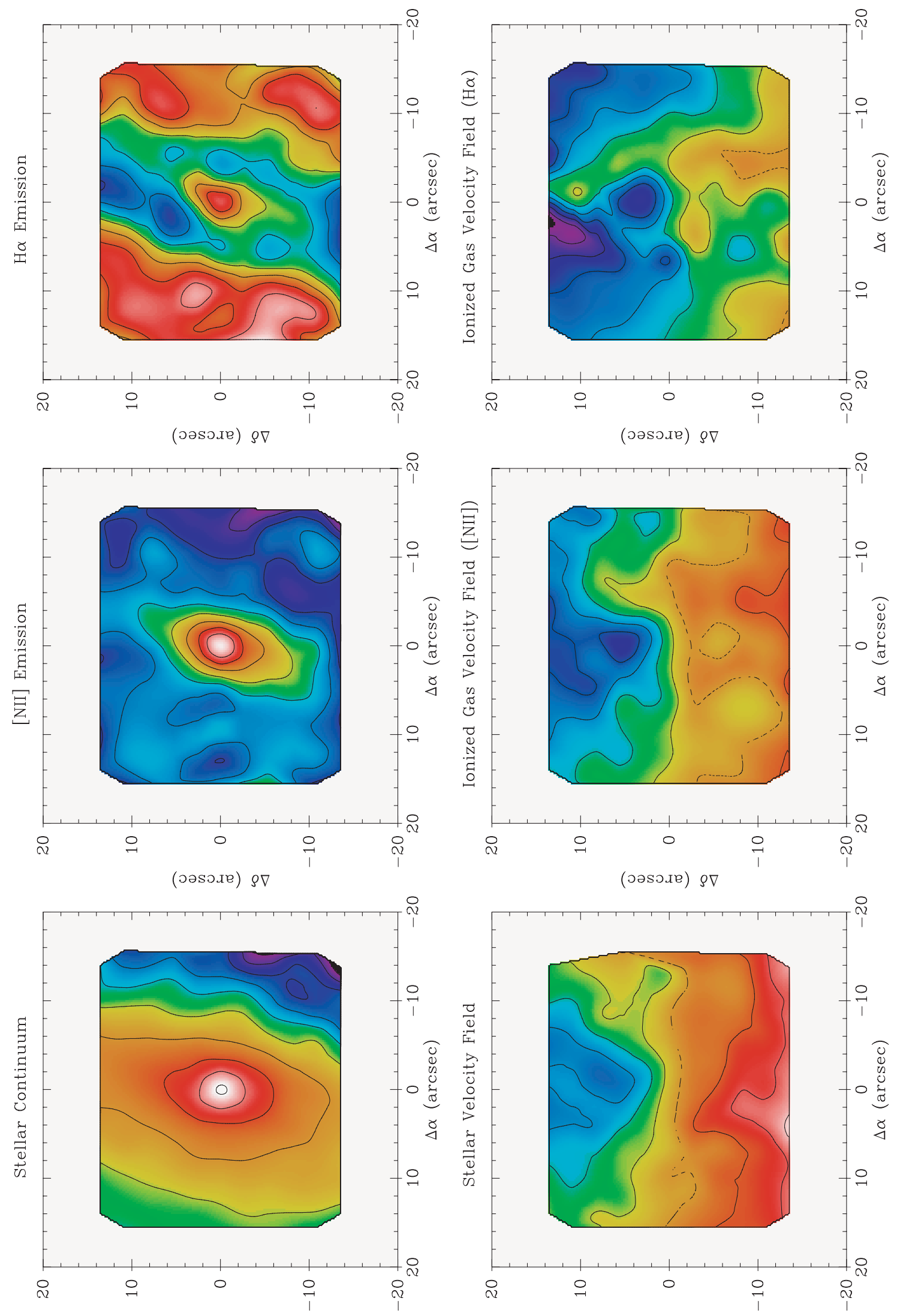

Fig. 1. Intensity and velocity maps for NGC 7331 (see text). The intensity maps isophotes (arbitrary units) are logaritmically scaled. The steps between consecutive isophotes are $0.31,0.18$, and 0.25 for the continuum, [NII] and $\mathrm{H} \alpha$ maps, respectively. The step between two consecutive iso-velocity lines is $35 \mathrm{~km} \mathrm{~s}^{-1}$. Approaching (receding) velocities correspond to blue (red) colors. The dashed isovelocity line corresponds to $v=830 \mathrm{~km} \mathrm{~s}^{-1}$, the systemic velocity according to Mediavilla et al. (1997). 


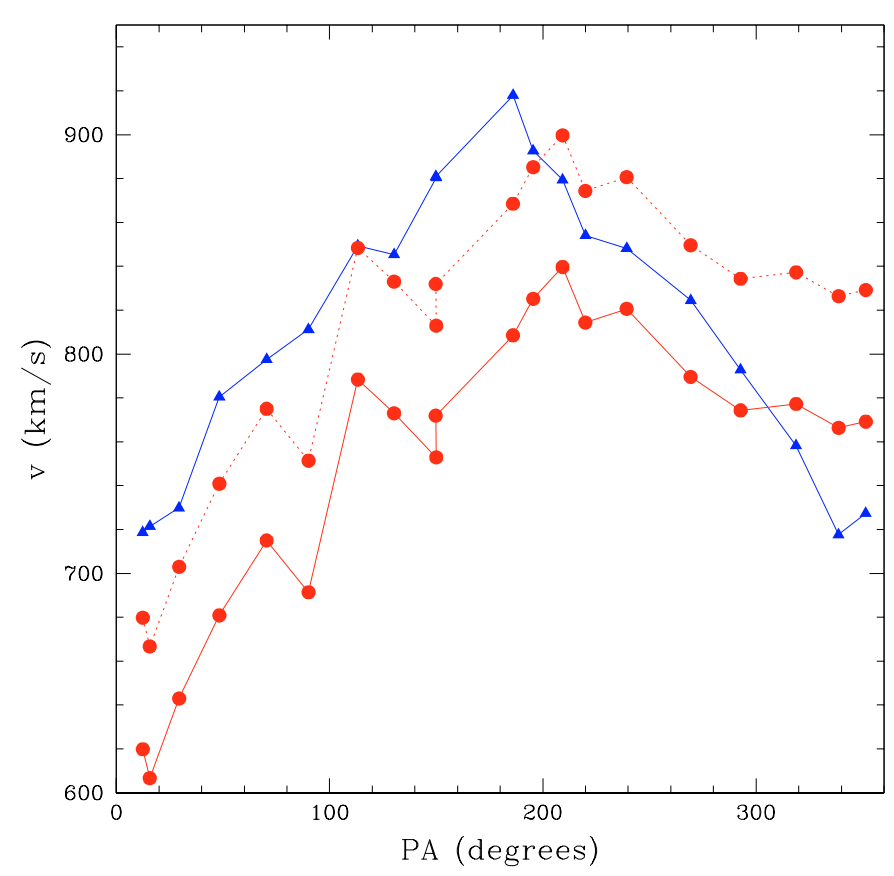

Fig. 2. $v(\theta)$ along the peculiar velocities ring. Triangles correspond to the stellar component. Circles joined by a solid line correspond to $\mathrm{H} \alpha$. Circles joined by a dot line correspond to $\mathrm{H} \alpha$ corrected from blueshift (see text).

blueshift in the framework of these alternative explanations. In any event, we favor axisymmetric inflow as the simplest explanation.

Regarding the global blueshift, it is noticeable that the magnitudes of the vertical (responsible of the global blueshift) and radial inward (causing the kinematic axis shifts) motions are in good quantitative agreement in both cases, $\mathrm{H} \alpha$ and [NII]. This suggests that we are seeing gas flowing from the edge of the $\mathrm{H} \alpha$ starburst ring in all allowed directions. Thus, the global blueshift is explained by the same process that gives rise to the radial inward motion.

There is good spatial coincidence between the peculiar velocities and $\mathrm{H} \alpha$ starburst rings (particularly good at the east region where the edge of the $\mathrm{H} \alpha$ ring is more sharply defined). Anyway, there is not significant differences in the results about the global blueshift and the radial inward motion at the galaxy plane if the velocity curves are determined along the $\mathrm{H} \alpha$ ring. The intermediate position of the [NII] kinematics between the stars and $\mathrm{H} \alpha$ can be explained if more than one kinematic component is contributing to the $[\mathrm{NII}]$ emission. This is supported by the existence of two-peaked [NII] lines (Mediavilla et al. 1997).

The inward velocity is much greater than the sound speed and a pressure gradient can be ruled out as the mechanism causing the gas inflow. However, it is of the order of the Alfven velocity if we consider a magnetic field of $10^{-5}$ Gauss. That means that the inflow of gas from the ring to the center could be induced by magnetic field pressure forces if a strong but in principle acceptable magnetic field were associated to the ring (see Battaner et al. 1988). Alternatively, the inflow can be also attributed to the presence of strong winds originated in

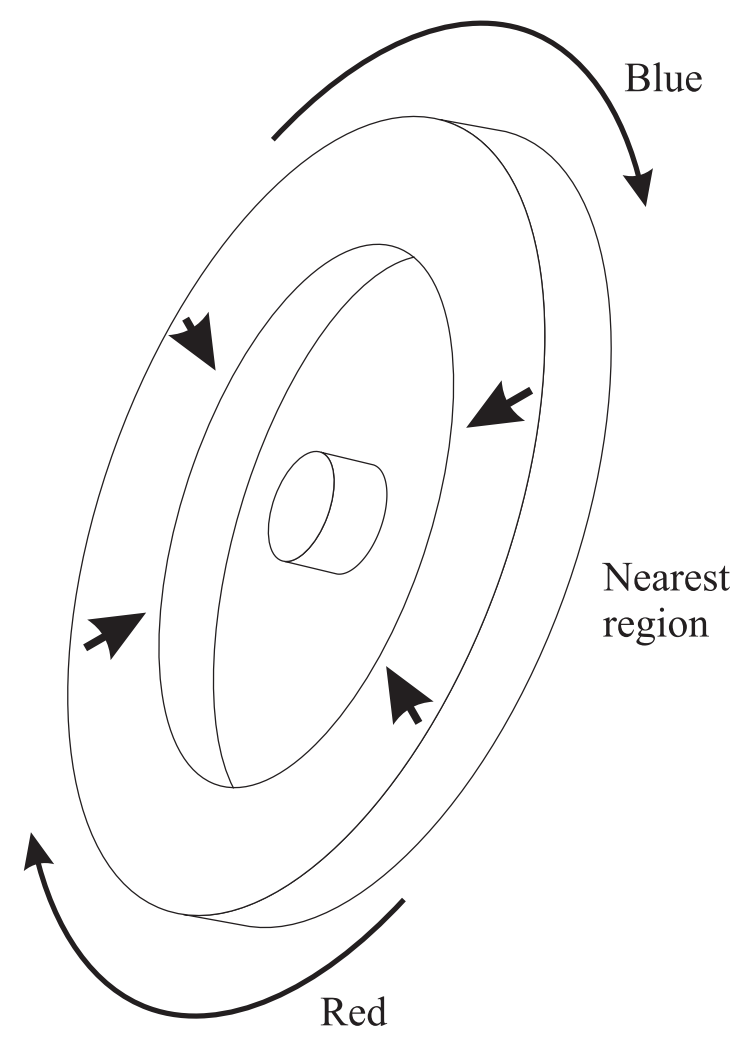

Fig. 3. A scheme of the nearly axisymmetric gas flow from the $\mathrm{H} \alpha$ ring to the central inner region detected in NGC7331. West is to the right and North is up. The internal radius of the plotted ring is about $2 \mathrm{kpc}$.

starbursts. The existence of a large scale process of stellar formation in NGC 7331 was suggested by Young \& Scoville (1982) to explain the presence of the CO ring in terms of exhaustion of the gas to form stars in the central region. The discovery of large scale stellar formation in the ring fits naturally in this hypothesis that is also supported by studies of the $\mathrm{Mg} 2$ index (del Burgo et al. 1997).

The gas inflow from the ring could eventually be accreted into the central black hole. To do an estimation of the accreted mass rate, we consider that the inwards flux is $2 \pi \mathrm{RHV} \rho$. The gas density $(\rho)$ is taken as $0.5 \times 10^{-2} M_{\odot} \mathrm{pc}^{-3}$ (Israel \& Baas 1999), the equivalent gas disc height as $H=467 \mathrm{pc}$ (Bottema 1999) and the galactocentric radius as $R=2 \mathrm{kpc}$. Therefore we estimate the infalling flux as $1.6 M_{\odot} \mathrm{yr}^{-1}$ of the order of magnitude of the accretion mass rate in a luminous seyfert nuclear black hole (Blandford et al. 1990).

For a low luminosity AGN, such as that in $\mathrm{M} 81$, the required accretion flux has been estimated to be of the order of $5 \times 10^{-5} M_{\odot} / y r$ (Ho et al. 1997), assuming an efficiency of conversion between matter and energy of 0.1 . As NGC 7331 has a nucleus of comparable luminosity (Stockdale et al. 1998) it seems reasonable that a part of the radial macroscopic infall observed here can feed the NGC 7331 AGN.

Considering the large difference between the scales of the nuclear accretion disc and the macroscopic ring observed here, the interpretation of the inward flux as the feeding of the central black hole can only be considered as tentative. Actually, this identification should be assessed under the conditions of 
continuity and stationarity. Even the inward velocity, as a function of radius, peaks at the inner ring boundary, it is not vanishing at lower radii. This fact favours the interpretation that the observed flux eventually reach the nucleus.

\section{Conclusions}

Some conclusions are worthy to be summarized:

We have detected an $\mathrm{H} \alpha$ ring coincident with the inner part of the well known $\mathrm{CO}$ and $\mathrm{HI}$ ring. The $[\mathrm{NII}] / \mathrm{H} \alpha$ ratio at the ring is typical of HII-like ionization and indicates that a large scale stellar formation process is taken place at the ring. This is in agreement with the hypothesis of that the gas in the inner to the ring region has been exhausted by massive stellar formation, that continues at the ring. The physical conditions in the inner to the ring region are typical of LINERS and the transition between starburst and LINER takes place very sharply at the inner boundary of the $\mathrm{H} \alpha$ ring.

Ionized gas peculiar velocities are found near to the inner boundary of the CO-HI ring, compatible with an axisymmetric morphology superimposed to the otherwise regular map of the ionized gas. The comparison with the regular stellar velocity map makes the assumption of an axisymmetric contraction very plausible. The infalling matter is observed at the inner boundary of the large-scale gaseous ring and likely continues towards the inner region. Although several mechanisms are possible to explain the origin of the inflow, the existence of strong winds at the ring wall fits naturally with the presence of massive stellar formation in the ring.

Even if the accreting flux could have very different causes we would be observing infalling velocities of the order of $50 \mathrm{~km} \mathrm{~s}^{-1}$ and infalling gaseous fluxes of the order of $1 M_{\odot} \mathrm{yr}^{-1}$. This is higher than the required accretion rate for a central black hole. Probably we are observing the infalling motion feeding the black hole having its source as far as $2 \mathrm{kpc}$ away from the center.

The inwards flux axisymmetry seems to rule out, in this galaxy, mechanisms based on bars. Bars have been proposed as sources for feeding AGN's and this have been observed to operate in the central regions of some active galaxies (See Perez et al. 2000, and references therein). NGC 7331, without a clearly observed bar, and with an axisymmetric inflow suggest that other ways of loosing angular momentum could also be important.

Acknowledgements. The Isaac Newton Group of Telescopes (ING) operates the $4.2 \mathrm{~m}$ William Herschel Telescope on behalf of the Particle Physics and Astronomy Research Council (PPARC) of the United Kingdom and the Netherlands Organization for Scientific Research (NWO) of the Netherlands. The ING is located at the Roque de Los Muchachos Observatory, La Palma, Spain.

This paper has been in part supported by the "Plan Andaluz de Investigación" (FQM-108) and by the "Secretaría de Estado de Política Científica y Tecnológica" (AYA2000-1574).

We acknowledge the improvement of the paper by an anonymous referee.

\section{References}

Afanasiev, V. L., Sil'chenko, O. K., \& Zasov, A. V. 1989, A\&A, 213, L9

Arribas, S., Mediavilla, E., del Burgo, C., \& García-Lorenzo, B. 1999, ApJ, 511, 680

Arribas, S., Carter, D., Cavaller, L., et al. 1998, SPIE, 3355, 821

Arribas, S., Mediavilla, E., Garcia-Lorenzo, B., \& del Burgo, C. 1997, ApJ, 490, 227

Arribas, S., Mediavilla, E., \& Rasilla, J. L. 1991, ApJ, 369, 260

Battaner, E., Florido, E., \& Sanchez-Saavedra, M. L. 1988, ApJ, 331, 116

Bingham, R. G., Gellatly, D. W., Jenkins, C. R., \& S Worswick, S. P. 1994, Proc. SPIE, 2198, 56

Blandford, R. D., Netzer, H., \& Woltjer, L. 1990, Saas-Fee Advanced Course, 20, Swiss Society for Astrophysics and Astronomy

Bosma, A. 1978, Ph.D. Thesis, Univ. of Groningen

Bottema, R. 1999, A\&A, 348, 77

Bower, G. A., Richstone, D. O., Bothun, G. D., \& Heckman, T. M. 1993, ApJ, 402, 76

del Burgo, C., Mediavilla, E., Arribas, S., \& Garcia-Lorenzo, B. 1997, Quasar hosts, Proceedings of the ESO-IAC Conference held on Tenerife, Spain, ed. D. L. Clements, \& I. Perez-Fournon, 171

Cowan, J. J., Romanishin, W., \& Branch, D. 1994, ApJ, 436, 139

Filippenko, A. V., \& Sargent, W. L. W. 1985, ApJS, 57, 503

García-Lorenzo, B., Arribas, S., \& Mediavilla, E. 2001, A\&A, 378, 787

García-Lorenzo, B., Mediavilla, E., \& Arribas, S. 1999, ApJ, 518, 190

Ho, L. C., Filippenko, A. V., \& Sargent, W. L. W. 1997, ApJ, 487, 591

Israel, F. P., \& Baas, F. 1999, A\&A, 351, 10

Marcelin, M., Petrosian, A. R., Amram, P., \& Boulesteix, J. 1994, A\&A, 282, 363

Mediavilla, E., Arribas, S., Garcia-Lorenzo, B., \& del Burgo, C. 1997, ApJ, 488, 682

Mediavilla, E., \& Arribas, S. 1993, Nature, 365, 420

Pérez, E., Márquez, I., Marrero, I., et al. 2000, A\&A, 353, 893

Prada, F., Gutierrez, C. M., Peletier, R. F., \& McKeith, C. D. 1996, ApJ, 458, 537

Stockdale, C. J., Romanishin, W., \& Cowan, J. J. 1998, ApJ, 508, 33

Telesco, C. M., Gatley, I., \& Stewart, J. M. 1982, ApJ, 263, 13

Tosaki, T., \& Shioya, Y. 1997, ApJ, 484, 664

von Linden, S., Reuter, H.-P., Heidt, J., Wielebinski, R., \& Pohl, M. 1996, A\&A, 315, 52

Young, J. S., \& Scoville, N. 1982, ApJ, 260, 41 